\title{
CRF-PEPICO: Double velocity map imaging photoelectron photoion coincidence spectroscopy for reaction kinetics studies
}

\author{
Bálint Sztáray, ${ }^{1, a)}$ Krisztina Voronova, ${ }^{1}$ Krisztián G. Torma, ${ }^{1}$ Kyle J. Covert, ${ }^{1}$ Andras Bodi, ${ }^{2}$ \\ Patrick Hemberger, ${ }^{2}$ Thomas Gerber, ${ }^{2}$ and David L. Osborn ${ }^{3, a)}$ \\ ${ }^{1}$ Department of Chemistry, University of the Pacific, Stockton, California 95211, USA \\ ${ }^{2}$ Laboratory for Femtochemistry and Synchrotron Radiation, Paul Scherrer Institute (PSI), \\ CH-5232 Villigen, Switzerland \\ ${ }^{3}$ Combustion Research Facility, Sandia National Laboratories, Livermore, California 94551, USA
}

(Received 2 March 2017; accepted 16 May 2017; published online 8 June 2017)

Photoelectron photoion coincidence (PEPICO) spectroscopy could become a powerful tool for the time-resolved study of multi-channel gas phase chemical reactions. Toward this goal, we have designed and tested electron and ion optics that form the core of a new PEPICO spectrometer, utilizing simultaneous velocity map imaging for both cations and electrons, while also achieving good cation mass resolution through space focusing. These optics are combined with a side-sampled, slow-flow chemical reactor for photolytic initiation of gas-phase chemical reactions. Together with a recent advance that dramatically increases the dynamic range in PEPICO spectroscopy [D. L. Osborn et al., J. Chem. Phys. 145, 164202 (2016)], the design described here demonstrates a complete prototype spectrometer and reactor interface to carry out time-resolved experiments. Combining dual velocity map imaging with cation space focusing yields tightly focused photoion images for translationally cold neutrals, while offering good mass resolution for thermal samples as well. The flexible optics design incorporates linear electric fields in the ionization region, surrounded by dual curved electric fields for velocity map imaging of ions and electrons. Furthermore, the design allows for a long extraction stage, which makes this the first PEPICO experiment to combine ion imaging with the unimolecular dissociation rate constant measurements of cations to detect and account for kinetic shifts. Four examples are shown to illustrate some capabilities of this new design. We recorded the threshold photoelectron spectrum of the propargyl and the iodomethyl radicals. While the former agrees well with a literature threshold photoelectron spectrum, we have succeeded in resolving the previously unobserved vibrational structure in the latter. We have also measured the bimolecular rate constant of the $\mathrm{CH}_{2} \mathrm{I}+\mathrm{O}_{2}$ reaction and observed its product, the smallest Criegee intermediate, $\mathrm{CH}_{2} \mathrm{OO}$. Finally, the second dissociative photoionization step of iodocyclohexane ions, the loss of ethylene from the cyclohexyl cation, is slow at threshold, as illustrated by the asymmetric threshold photoionization time-of-flight distributions. Published by AIP Publishing. [http://dx.doi.org/10.1063/1.4984304]

\section{INTRODUCTION}

\section{A. Background}

Photoionization mass spectrometry (PIMS) ${ }^{1-4}$ is a universal, sensitive, selective, and multiplexed analytical technique to interrogate chemical reactions and has been very productive at tunable vacuum ultraviolet synchrotron light sources ${ }^{5}$ in combustion studies, ${ }^{6}$ among numerous other fields. ${ }^{7}$ At the Chemical Dynamics Beamline of the Advanced Light Source, the time-resolved multiplexed photoionization mass spectrometer (MPIMS) ${ }^{5}$ has detected and enabled the study of longsought reactive intermediates important in combustion ${ }^{8,9}$ and atmospheric ${ }^{10,11}$ chemistry, and has provided isomer-resolved product studies of chemistry relevant to astrochemical environments ${ }^{12-14}$ including Titan, Saturn's largest moon. ${ }^{15}$ More

\footnotetext{
a) Authors to whom correspondence should be addressed: bsztaray@ pacific.edu and dlosbor@sandia.gov
}

specifically, it enables the study of bimolecular chemical reactions by observing all reactants and products simultaneously, with enough dynamic range $\left(10^{5}\right)$ to detect minor concentrations of key reactive intermediates in the presence of other species at much higher concentrations. However, the ability of PIMS to distinguish different isomers detected at the same mass-to-charge ratio $(\mathrm{m} / \mathrm{z})$ relies on the ionization energies and shapes of their photoionization spectra. It would be a major improvement to acquire more detailed spectral fingerprints of neutral species, while maintaining all the other advantages of PIMS. Photoelectron spectroscopy is a promising approach to meet this goal, but without mass selection of the corresponding cations, it is ill-suited to analyze complex mixtures. Photoelectron photoion coincidence (PEPICO) spectroscopy, which requires the detection of photoions and photoelectrons in delayed coincidence, enabling the mass analysis of the former and the kinetic energy analysis of the latter, could offer the best of both worlds. ${ }^{16}$ PEPICO has already been used to identify and characterize pyrolysis products, ${ }^{17-19}$ explore thermal decomposition ${ }^{20}$ and even bimolecular 
processes, ${ }^{21}$ sometimes explicitly complementing PIMS and other spectroscopic approaches. ${ }^{22}$

A decade ago, PEPICO faced four challenges to its widespread use for the chemical analysis of reacting systems. ${ }^{23}$ Three of these challenges have recently been overcome, and the fourth is addressed in this paper. First, where sensitivity and spectral resolution were previously lacking, threshold electron detection now offers the combination of a favorable energy resolution (less than $1 \mathrm{meV}$ ) and enhanced collection efficiency, thanks to the introduction of velocity map imaging (VMI), ${ }^{24,25}$ in particular, when combined with fast delay line detectors in imaging photoelectron photoion coincidence (iPEPICO) experiments. ${ }^{26,27}$ Second, full utilization of the high photon flux available at modern light sources led to the creation of new data acquisition and analysis approaches to avoid signal paralysis. ${ }^{28}$ This new capability for high-throughput experiments brought to the surface a third challenge: the limited dynamic range of PEPICO. A photoelectron-photoion pair arising from two separate ionization events, but arriving in the time-of-flight (TOF) window of interest, is termed a false coincidence. At high ionization rates, false coincidences create a constant background in the TOF mass spectrum with random noise around the average background value. While the average false coincidence background can be subtracted from the TOF spectrum, its Poisson scatter can obscure minor peaks and limits the dynamic range to about $10^{3}$ within a reasonable integration time. This is a major issue in analytical applications, especially for detection of trace species, where a high dynamic range is essential. To address this limitation, we conceived and tested a new method that deflects photoions by a periodically changing but known electric field, applied at a constant fraction of each photoion's time of flight. For a true coincidence, i.e., an electron-ion pair arising from one neutral species, the measured and predicted ion impact positions agree. If the electron-ion pair is a false coincidence, the assumed TOF is spurious, and the predicted impact position of the ion on the detector does not match its measured position. Having identified this coincidence event as false, it can be removed from the dataset. The almost quantitative elimination of the false coincidence background increases the dynamic range dramatically, from $10^{3}$ to at least $10^{5}-10^{6}$, which is sufficient for most analytical applications. ${ }^{29}$ The fourth challenge relates to mass resolution in the TOF spectrum. The MPIMS approach, which detects only cations, achieves a mass resolution of $m / \Delta m \approx 1500,{ }^{30}$ sufficient to separate nearly isobaric species such as $\mathrm{HCO}^{+}$from $\mathrm{C}_{2} \mathrm{H}_{5}{ }^{+31}$ Although a mass resolution of $\approx 500$ suffices for most applications, it remains a challenge to combine such performance, which benefits from high extraction fields, with coincident high resolution photoelectron kinetic energy analysis, which requires low extraction fields. The present paper describes a prototype experiment combining advances in all four areas, which we call Combustion Reactions Followed by Photoelectron Photoion Coincidence (CRF-PEPICO). It combines simultaneous velocity map imaging of photoelectron and photoions in coincidence with good cation mass resolution achieved by Wiley-McLaren space focusing.

The flexible implementation of the electron and ion optics also allowed a further, equally new feature of this apparatus, namely, adding a slow-flow laser photolysis reactor tube in the immediate vicinity of the ionization region. We have thus been able to build a kinetic time-resolved PEPICO experiment to study gas phase reacting systems, while also improving the ability to study the photoionization process itself, including the measurement of unimolecular rate constants in dissociative photoionization.

\section{B. A new approach to double imaging}

Our method to distinguish true and false coincidences ${ }^{29}$ requires measuring the deflection of cations in the $x$ and $y$ axes perpendicular to the TOF axis ( $z$ axis), and therefore we must use an imaging detector for the cations. The use of imaging has recently been incorporated at three synchrotron-based continuous imaging PEPICO experiments around the world, and we briefly describe these different approaches to place our new apparatus in context.

The coincidence experiment at the Hefei National Synchrotron Radiation Laboratory records ion velocity map images using a CCD camera, which is too slow for the simultaneous detection of the mass spectrum and thus requires time slicing. ${ }^{32}$ While this approach directly detects the equatorial region of the Newton spheres, making image reconstruction unnecessary, better signal-to-noise ratios could be obtained by recording the complete VMI projection and reconstructing the momentum distribution of the ions. Nevertheless, the Hefei PEPICO experiment is fully capable of recording at least unit resolution threshold ionization mass spectra for translationally cold species.

The DELICIOUS III endstation at the SOLEIL synchrotron uses two fast delay-line detectors, which enable continuous acquisition of the complete mass spectrum. ${ }^{33}$ The electrons are velocity map imaged onto the electron detector; however, VMI is not used for the cations. Instead, by using a low extraction field, any kinetic energy imparted to the cation from photoionization or photofragmentation leads to a Newton sphere that has expanded significantly in all 3 dimensions by the time the cation impacts the detector. From the $(x, y)$ spatial coordinates of ion impact at the detector and the TOF (which carries the $z$-component velocity information) of each cation, the full 3-D momentum vector of the cation may be extracted on an event-by-event basis. No reconstruction of the image is needed to extract this information, the polarization of the ionizing radiation may be freely chosen, and a $100 \%$ duty cycle is achieved using fast detectors. However, the low extraction field means that high mass resolution can only be attained for ions translationally cold along the $z$-axis axis. If the sample is translationally warm or the fragment ion obtains kinetic energy upon dissociation, the mass resolution decays quickly. The SOLEIL experiment has recently been updated to include double skimming of the molecular beam to ensure translationally cold samples. ${ }^{34}$

The third existing instrument, the $i^{2}$ PEPICO endstation at the Swiss Light Source, utilizes symmetric VMI for both electrons and cations. ${ }^{35}$ Using VMI, the Newton sphere is "pancaked" at the detector, so that the velocity components perpendicular to the TOF axis are projected onto the detector face, with minimal blurring from spatial spreads in the 
ionization position. However, the effect of the $z$-axis momentum component on the TOF is almost negligible, so that the 3-D momentum vector cannot be directly measured for each individual cation. Nevertheless, the 3-D momentum distribution of a group of cations can be reconstructed from the cation image so long as the linearly polarized electric vector of the ionizing radiation is parallel to the plane of the detector. Although one would like to obtain the correlation of the photoelectrons with their coincident mass-analyzed cation, such a double VMI inversion approach has not yet been demonstrated. Two less comprehensive data treatments are possible instead. Velocity map images of electrons detected in coincidence with a cation at a particular $\mathrm{m} / \mathrm{z}$ ratio can be reconstructed to obtain photoion mass selected photoelectron spectra. Alternatively, the subtraction procedure proposed by Sztáray and Baer $^{25}$ and its more advanced version utilizing the whole photoelectron velocity map image ${ }^{36}$ can be applied to obtain the velocity map image of energy-selected photoions and to reconstruct the 3-D momentum distribution belonging to a certain $\mathrm{m} / \mathrm{z}$ channel. However, the ion optics of the $i^{2}$ PEPICO apparatus do not employ strict Wiley-McLaren space focusing, and hence provide a mass resolution of only about $m / \Delta m \approx$ 100-150 in room temperature samples at a relatively high extraction field of $120 \mathrm{~V} \mathrm{~cm}^{-1}$. This moderate mass resolution indirectly causes another disadvantage: a wide peak results in a low signal-to-background ratio with respect to the false coincidence background, further limiting the dynamic range.

To address the challenges described above, we had three main design objectives for the CRF-PEPICO instrument. First, we sought a mass resolving power of at least 400-500, even for photoionized room-temperature parent neutrals, or when kinetic energy is imparted to a daughter ion via dissociation. Second, we sought the ability to study time-resolved kinetics of reacting systems of neutrals, initiated by laser photolysis that creates reactive free radicals. Third, none of the previously existing double imaging experiments can readily measure unimolecular rate constants for the decay of metastable cations. These rate constants are needed to determine dissociative photoionization onsets for use in high precision thermochemistry. To achieve all three goals, our design contains a linear extraction field at the point of ionization, surrounded on both the electron and cation sides by higher electric field regions that incorporate curvature in the equipotential lines, enabling independent velocity map imaging for electrons and cations. The two electric field strengths meet the extraction and acceleration field criteria of Wiley-McLaren space focusing, ${ }^{37}$ which improves mass resolution for spatially extended neutral sources.

Our false coincidence rejection technique requires tight ion focusing by VMI, which also results in sharp momentum imaging of the photoions in the plane perpendicular to the extraction axis. To the best of our knowledge, the required combination of space focusing and velocity map imaging conditions had not been achieved simultaneously. The flexibility to vary the extraction field between 20 and $250 \mathrm{~V} \mathrm{~cm}^{-1}$ is desirable to change the energy range fully imaged onto the detector and to vary the range of measurable ion dissociation rate constants. Therefore, we required flexibility in two parameters: extraction field strength and extraction region length.

\section{EXPERIMENTAL}

The ion and electron optics were designed to accommodate a side-sampled, quartz flow-tube reactor, pioneered by Gutman et al. ${ }^{38}$ It is placed within the ion optics to minimize the distance from the pinhole in the reactor tube and the ionization point. The challenge of incorporating a non-conducting quartz tube near the ionization point, together with the goals of dual VMI and space focusing discussed above led us to build a prototype experiment before attempting to build an entirely new apparatus. To test the new design, we utilized most of the $i^{2}$ PEPICO infrastructure at the vacuum ultraviolet (VUV) beamline of the Swiss Light Source. The prototype is also compatible with other sample inlet systems at this beamline, including a supersonic molecular beam source, a microtubular pyrolysis reactor, a pulsed catalytic reactor setup, and a low pressure flame experiment.

The VUV synchrotron radiation is linearly polarized in the horizontal direction and is less than $3 \mathrm{~mm}$ in height and less than $6 \mathrm{~mm}$ in width at the ionization point. The sample gas absorbs the VUV light, forming photoelectrons and photoions which are extracted vertically in opposite directions by a linear electric field, accelerated and focused by the multi-plate charged particle optics (vide infra). After passing through field-free regions, the charged particles are detected by two Roentdek DLD40 position sensitive fast delay-line detectors. The $x_{1}, x_{2}, y_{1}$, and $y_{2}$ delay-line signals from both detectors are digitized, together with a trigger signal from the photolysis laser when it is used. The measurement program records the electron and ion event detection times and positions, as well as the trigger timestamps. Electron-ion coincidence events and their timing with respect to the trigger (laser) pulse can be analyzed post-measurement, based on the regions of interest on both position sensitive detectors, time elapsed from the trigger signal, and the time of flight of the ion. $^{35}$

\section{A. Electron and ion optics}

Velocity map imaging requires gridless electron and ion optics, generating curved focusing fields, whereas space focusing ${ }^{37}$ normally relies on well-defined first and second acceleration regions with homogeneous (linear) electric fields enabled by semi-transparent conducting grids. These opposing requirements make it challenging to combine space focusing with VMI. To forgo the use of grids and maximize the adaptability of the electron and ion optics, we use a large number of individually controllable electrode plates, 12 on the electron side and 14 on the ion side (see Fig. 1), similar to the Hatom VMI experiment by Ryazanov and Reisler. ${ }^{39}$ The plates are of $24 \mathrm{~mm}$ inner diameter and $90 \mathrm{~mm}$ outer diameter, 0.5 $\mathrm{mm}$ thick, each separated by $6.25 \mathrm{~mm}$ with the exception of the final seven plates on the ion side, where the spacing increases to $9 \mathrm{~mm}$. The extraction field at the ionization point can be varied between 20 and $250 \mathrm{~V} \mathrm{~cm}^{-1}$. The first two plates around the ionization region are circular segments that have been cut along a chord to accommodate the flow 


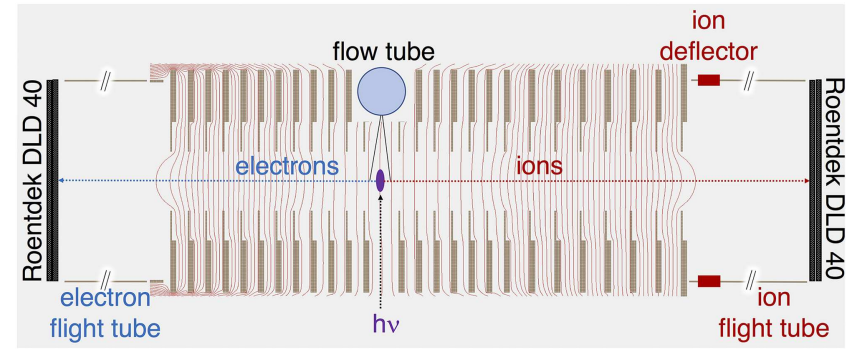

FIG. 1. Section view along the centerline of the individually controllable electrode plates of the electron (left) and ion (right) sides with calculated equipotential lines.

tube that runs between the second ion and electron plates. The potential at the ionization point is nominally at ground. The effective length of the Wiley-McLaren extraction (lowfield) and acceleration (high-field) regions, providing space focusing and VMI, can be varied without breaking vacuum by individually setting the voltages on the electrode plates, whereas the total length of the two regions is established by setting the remaining plates (if any) to the flight tube voltage. The total lengths of the extraction/acceleration/VMI optics are 75.5 and $105.7 \mathrm{~mm}$ on the electron and ion sides, respectively. The field free electron flight tube is a $62 \mathrm{~mm}$ I.D. $\mu$-metal cylinder, and the detector is mounted $750 \mathrm{~mm}$ from the ionization point. On the ion side, the $60 \mathrm{~mm}$ I.D. flight tube houses the ion deflector needed for the false coincidence rejection, ${ }^{29}$ which was not used in the experiments reported here. The ion detector face is $1000 \mathrm{~mm}$ from the ionization point.

\section{B. Flow tube}

The component gases are metered by mass flow controllers and flow in a side-sampled, slow-flow reactor, as described by Osborn et al. ${ }^{5}$ The reactor is a $60 \mathrm{~cm}$ long quartz tube with $1.27 \mathrm{~cm}$ outer diameter, $1.05 \mathrm{~cm}$ inner diameter, and a $300 \mu \mathrm{m}$ pinhole at the halfway-point along the tube. The pressure in the reactor tube is maintained between 1 and 4 mbar. The $300 \mu \mathrm{m}$ pinhole emits a gas mixture toward the ionization region, where the background pressure is kept below $6 \times 10^{-6}$ mbar without the need for additional differential pumping. A $20 \mathrm{~Hz}$ pulsed Nd-YAG laser beam at $\lambda=355$ or $266 \mathrm{~nm}$, with a pulse energy of 80 or $30 \mathrm{~mJ}$, respectively, propagates collinearly inside the reactor tube, photodissociating precursor molecules to create free radicals. The total gas flow rate is sufficient to replace the gas mixture in the tube with a fresh sample before the next laser pulse. The pressure inside the tube is sufficiently low that the spatial distribution of radicals becomes radially and axially homogeneous on a time scale that is short compared to the kinetics of the gasphase bimolecular reactions. The inner surface of the quartz tube is coated with halocarbon wax to minimize radical loss on the reactor wall. Minimizing the distance between the gasemitting pinhole in the reactor wall and the ionization volume maximizes sensitivity. Therefore, as mentioned before, the two electrode plates surrounding the ionization point are cut along a chord $26 \mathrm{~mm}$ from their edge, enabling a pinhole to ionization point distance that is variable between $21 \pm 4 \mathrm{~mm}$. The centerline of the flow tube is parallel to the synchrotron radiation beam.

\section{RESULTS}

\section{A. Electron and ion VMI, mass resolution}

Photoelectrons are velocity map imaged onto the positionsensitive delay-line detector. The maximum perpendicular velocity component that falls on the active detector area can be controlled by either the extraction field strength or by changing the relative lengths of the extraction and acceleration regions and adjusting the latter electric field to satisfy the VMI conditions. This approach allows flexibility to adjust the range of imaged photoelectron kinetic energies while maintaining good mass resolution on the ion side. A photoelectron velocity map image of argon at $h v=16 \mathrm{eV}$ and $125 \mathrm{~V} \mathrm{~cm}^{-1}$ extraction field is shown in Fig. 2, together with the reconstructed photoelectron spectrum (PES). The image is somewhat affected by stray magnetic fields and/or electric field penetration. This distortion was only taken into account in first order by approximating the Newton sphere projections as mildly eccentric ellipses. Without fine control to adjust the tilt in the optics, the focal

\section{(a)}

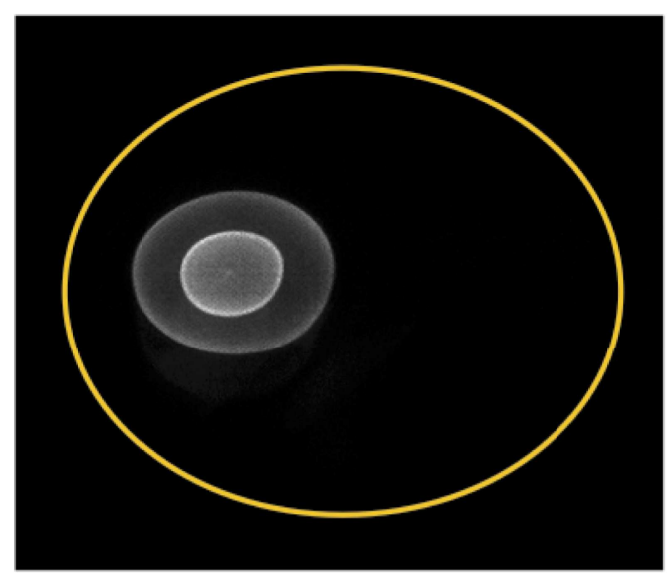

(b)

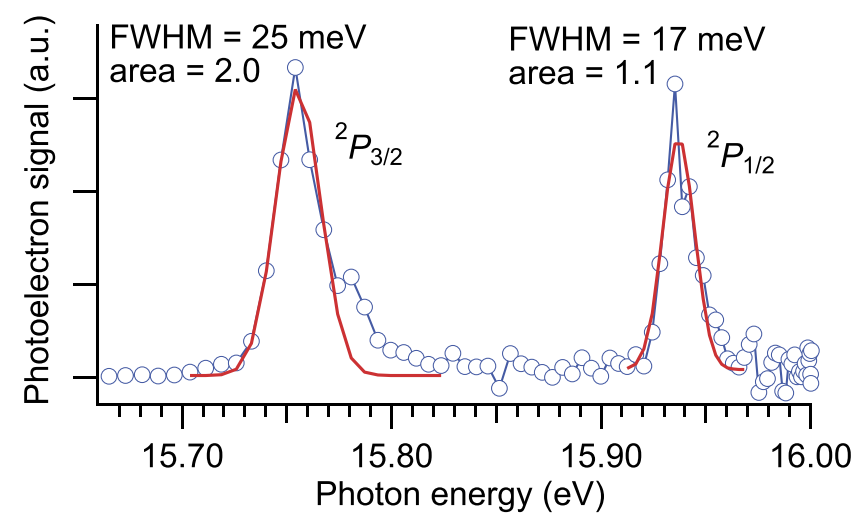

FIG. 2. A raw argon photoelectron velocity map image at a photon energy of $16 \mathrm{eV}$ is shown in (a) together with the approximate edge of the detector. The reconstructed photoelectron spectrum is seen in (b) together with Gaussian fits to both spin-orbit peaks. The photon energy resolution and the Stark shift contribute to a combined peak broadening of ca. 2-3 meV. 
point of the image is off-center, and the ca. $10 \%$ energy resolution, obtained at one-fourth of the detector energy range of $1.1 \mathrm{eV}$, implies better than 5\% energy resolution if the full detector area were used. Threshold electrons are focused to a spot with a radius that corresponds to less than $1 \mathrm{meV}$ kinetic energy.

As the equipotential lines in Fig. 1 demonstrate, the individually addressable multi-plate ion optics provide nearly linear extraction and appropriately curved fields to accomplish both space focusing and VMI. Figure 3 shows a mass

(a)

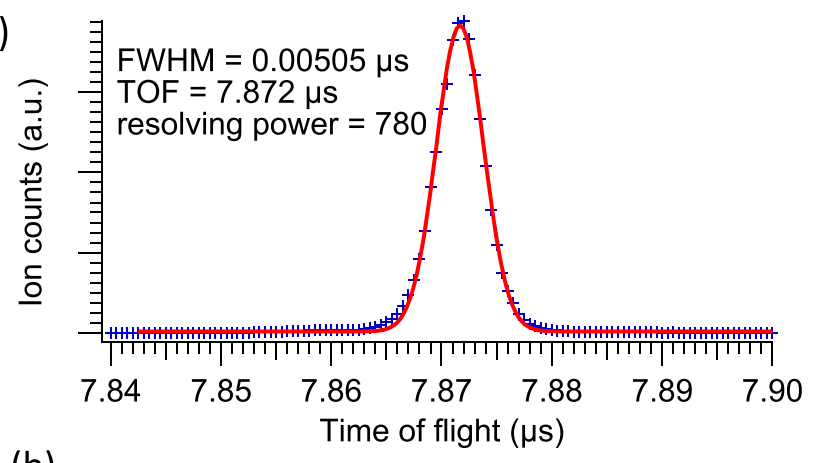

(b)

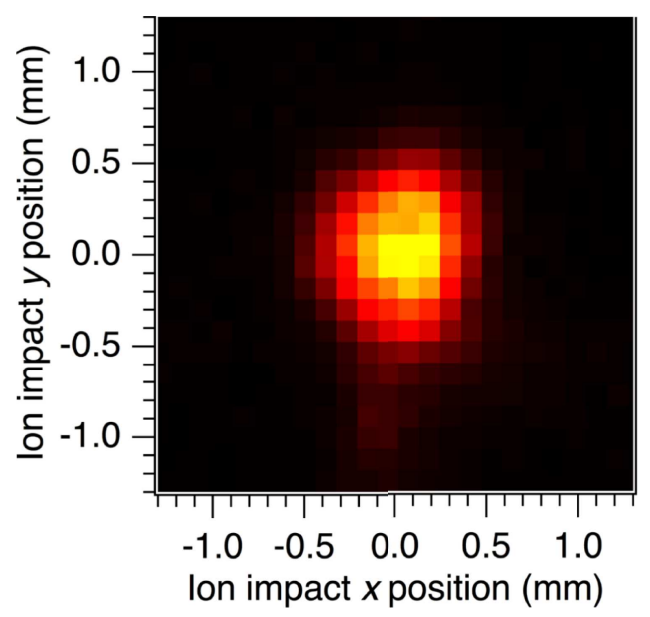

(c)

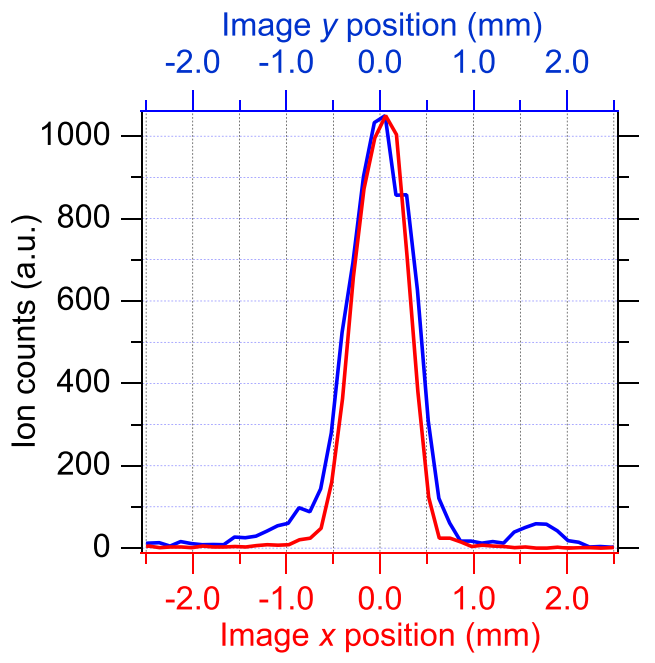

FIG. 3. (a) Ar time-of-flight mass spectrum. (b) Ar ion image using a molecular beam. (c) Cross sections of the focused ion spot, showing typical FWHMs of $0.7-0.8 \mathrm{~mm}$. resolution of 780 using the supersonic Ar molecular beam source and a tightly focused ion spot that is less than 0.7$0.8 \mathrm{~mm}$ FWHM on the detector, as reported in the falsecoincidence suppression paper. ${ }^{29}$ The ion velocity map image of an expansion from the low-pressure flow tube, however, exhibits a broader velocity distribution that will be discussed in Sec. III C.

\section{B. Threshold photoelectron spectrum of the propargyl radical}

Small, resonance-stabilized hydrocarbon radicals, such as the propargyl radical, $\mathrm{H}_{2} \mathrm{CCCH}$, are among the most abundant free radicals in rich hydrocarbon flames and may be present in planetary atmospheres. The self-reaction of propargyl radicals yields various $\mathrm{C}_{6} \mathrm{H}_{6}$ isomers, including benzene, and is particularly important in the formation of the first aromatic ring, considered to be the rate determining step in polycyclic aromatic hydrocarbon (PAH) growth. ${ }^{40}$ This chemistry has motivated numerous experimental ${ }^{41,42}$ and computational ${ }^{43,44}$ studies on its recombination kinetics, as well as on methods for propargyl detection and quantification. ${ }^{30,45-47}$ Hemberger et al. produced the propargyl radical by the flash pyrolysis of propargyl bromide, ionized it using VUV synchrotron radiation, and measured its photoion mass-selected threshold photoelectron spectrum (ms-TPES). ${ }^{47}$

In this work, we generated propargyl radicals by photodissociation at $266 \mathrm{~nm}$ of propargyl bromide diluted in argon. The measurement was conducted with an extraction field of $125 \mathrm{~V} \mathrm{~cm}^{-1}$, applied using 7 plates on the electron side and 4 plates on the ion side. The VMI regions consisted of an additional 5 plates on the electron side and 10 plates on the ion side. Photoelectrons were detected within 50 $\pm 0.4 \mathrm{~ns}$ of ionization, while the ion time of flight for $\mathrm{m} / \mathrm{z}$ $=39$ was $10.52 \mu \mathrm{s}$. As detailed above, a group of coincidence events can be selected over restricted ranges of ion and electron velocities, time of flight, i.e., photoion $\mathrm{m} / \mathrm{z}$, and kinetic times. Even at photon energies below the ionization energy (IE) of the precursor propargyl bromide $\left(\mathrm{C}_{3} \mathrm{H}_{3} \mathrm{Br}\right.$, IE $=10.42 \mathrm{eV}),{ }^{48}$ small amounts of scattered light ionized the precursor, which was present in great excess, making photoion mass selection essential to obtain a clean threshold photoelectron spectrum (TPES). To obtain the mass-selected TPES, shown in Fig. 4, coincidence events of threshold electrons ${ }^{25}$ and $m / z=39$ ions were plotted against the photon energy. The spectra were normalized by the photon flux as a function of photon energy. We corrected the spectrum for a Stark shift of $67 \mathrm{~cm}^{-1}$ (ca. $\left.8 \mathrm{meV}\right),{ }^{49}$ induced by the continuous extraction field of $125 \mathrm{~V} \mathrm{~cm}^{-1}$.

The ms-TPES of the $\mathrm{H}_{2} \mathrm{CCCH}$ radical in Fig. 4 is shown together with the spectrum measured by Hemberger et al. ${ }^{47}$ The single intense peak suggests a small geometry change upon ionization and can be assigned as the $0 \leftarrow 0$ transition. Therefore, its maximum at $8.705 \pm 0.010 \mathrm{eV}$ corresponds to the adiabatic ionization energy (AIE), in good agreement with the definitive value of $8.70053 \pm 0.00026 \mathrm{eV}$, obtained with zero kinetic energy photoelectron spectroscopy by Merkt et al. ${ }^{50}$ 


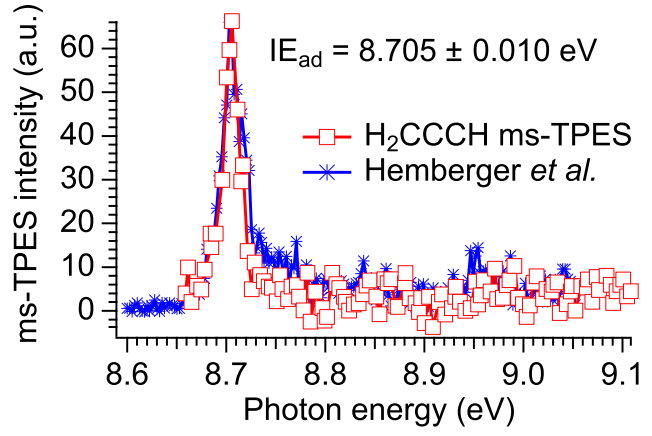

FIG. 4. Photoion mass-selected threshold photoelectron spectra of the propargyl radical, generated by photodissociation (red, this work) or pyrolysis (blue, Ref. 47) of $\mathrm{C}_{3} \mathrm{H}_{3} \mathrm{Br}$.

\section{C. lodomethyl TPES and $\mathrm{CH}_{2} \mathrm{I}+\mathrm{O}_{2}$ reaction kinetics}

Mono- and polyhalogenated iodine-containing organic compounds are the main source of iodine in the atmosphere through volatilization from the ocean. ${ }^{51}$ The simultaneous presence of UV radiation, iodocarbons, and ozone leads to homogeneous nucleation and aerosol formation. In particular, diiodomethane is an abundant iodine-containing compound, which reacts with ozone to produce $\mathrm{IO}$ and $\mathrm{CH}_{2} \mathrm{I}$ radicals under atmospheric conditions. The iodomethyl radical will then react with molecular oxygen, yielding the simplest Criegee-intermediate, $\mathrm{CH}_{2} \mathrm{OO}$, and then react with a second iodine atom, which may ultimately yield a further IO radical, a key intermediate in particle formation. ${ }^{52}$ The photoelectron spectrum of the iodomethyl radical was studied by Andrews et al. ${ }^{53}$ who observed a featureless vibrational envelope for the ground electronic state of the cation and reported an adiabatic IE of $8.40 \pm 0.03 \mathrm{eV}$ and a vertical IE of 8.53 $\pm 0.03 \mathrm{eV}$.

We used the $\mathrm{CH}_{2} \mathrm{I}+\mathrm{O}_{2}$ reaction to test the time-resolved capabilities of our prototype CRF-PEPICO spectrometer. Metered flows of $\mathrm{CH}_{2} \mathrm{I}_{2}$ and $\mathrm{O}_{2}$ diluted in argon were mixed in situ at the entrance of the flow tube, and the reaction was initiated by laser photolysis of $\mathrm{CH}_{2} \mathrm{I}_{2}$ at $355 \mathrm{~nm}$, producing $\mathrm{CH}_{2} \mathrm{I}$ radicals and $\mathrm{I}$ atoms. The decay of the $\mathrm{CH}_{2} \mathrm{I}$ radical was first monitored without the addition of $\mathrm{O}_{2}$ to obtain a wall-loss reaction rate coefficient of $\mathrm{CH}_{2} \mathrm{I}$ of $k_{\text {wall }}$ $=3 \pm 4 \mathrm{~s}^{-1}$. Simultaneously, we scanned the VUV photon energy and recorded the ms-TPES spectrum of the $\mathrm{CH}_{2} \mathrm{I}$ radical, as shown in Fig. 5. Extraction fields of 125 and $250 \mathrm{~V} \mathrm{~cm}^{-1}$ were used, and the ms-TPES was corrected for the Stark shift.

The ms-TPES exhibits a vibrational progression starting at $8.333 \mathrm{eV}$ with a spacing of $88 \mathrm{meV}$ (or $710 \mathrm{~cm}^{-1}$ ). B3LYP calculations using the 6-311++G(d,p) basis set on $\mathrm{C}$ and $\mathrm{H}$ and aug-cc-pVTZ-PP on I predict an AIE of $8.43 \mathrm{eV}$, and a $0.1 \AA$ reduction of the $\mathrm{C}-\mathrm{I}$ bond length upon ionization, exciting the C-I stretching vibration $\left(v_{3}{ }^{+}=751 \mathrm{~cm}^{-1}, \mathrm{~A}_{1}\right)$, consistent with the observed vibrational progression. To compare with the experimental ms-TPES, we calculated the room temperature photoelectron spectrum using the Franck-Condon (FC) approach as implemented in the program eZspectrum (Fig. 5) ${ }^{54}$ After shifting the calculated spectrum to match our assigned origin transition at $8.333 \mathrm{eV}$, the simulation

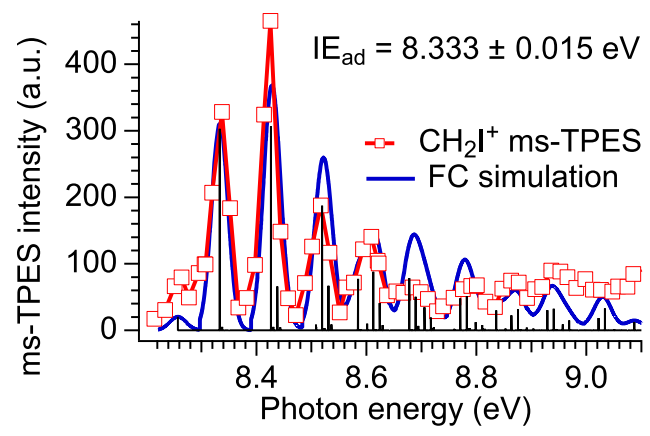

FIG. 5. Mass-selected threshold photoelectron spectrum of the $\mathrm{CH}_{2} \mathrm{I}$ radical along with the Franck-Condon simulation. Black sticks show computed transitions and their intensities. The blue line is obtained by convolving the transitions with a Gaussian function of $35 \mathrm{meV}$ FWHM to account for the rotational envelope.

reproduces the experimental spectrum well, with only minor differences in the relative intensities of the higher energy and hot band transitions. As expected from the geometry change, the $\mathrm{C}-\mathrm{I}$ stretching vibration is responsible for the major progression, which is accompanied and broadened by less intense transitions.

The photoelectron spectrum cannot be reproduced without contributions from the non-totally symmetric $\mathrm{H}-\mathrm{C}-\mathrm{H}$ neutral wagging mode, which, at $v_{4}=169 \mathrm{~cm}^{-1}\left(\mathrm{~B}_{1}\right)$, is excited at room temperature. Transitions in this mode yield heavily shifted sequence bands, because the corresponding $v_{4}{ }^{+}$mode in the cation has a much higher frequency at $1013 \mathrm{~cm}^{-1}$. The large change in this vibrational frequency combined with little geometry change upon ionization also means that the nuclear wave function overlap remains favorable for large changes in the excitation level. In addition, the simulation also includes combination bands with the $v_{3}{ }^{+}$mode, $1 v_{4} \rightarrow n v_{4}{ }^{+} \mathrm{mv}_{3}{ }^{+}$, and even quanta transitions from the neutral ground state into $(2 n) v_{4}{ }^{+}$, which play a crucial role in the simulation, owing to the relatively large excited $v_{4}$ population at room temperature. The vibrational structure in the PES of Andrews et $a l .{ }^{53}$ was probably missing because they used highly exothermic fluorine abstraction to prepare the iodomethyl radical. With an even larger excitation in the low-frequency $v_{4}$ of the neutral than at room temperature, the numerous FC-favored transitions may have smeared out all vibrational structures. The vibrational analysis of our room temperature ms-TPES lets us revise the adiabatic ionization energy to 8.333 $\pm 0.015 \mathrm{eV}$.

The concentration of $\mathrm{CH}_{2} \mathrm{I}_{2}$ was reduced until the $\mathrm{CH}_{2} \mathrm{I}$ decay, as monitored by the kinetic time trace of the $\mathrm{CH}_{2} \mathrm{I}^{+}$signal, showed no further dependence on $\left[\mathrm{CH}_{2} \mathrm{I}_{2}\right]$, as shown in Fig. 6(a). Note that the steeper decay after $20 \mathrm{~ms}$ is due to the bulk flow bringing fresh, un-photolyzed gas to the sampling pinhole. On the basis of the literature absorption spectrum of $\mathrm{CH}_{2} \mathrm{I}_{2} 55$ and the laser fluence of $125 \mathrm{~mJ} \mathrm{~cm}^{-2}$, the initial iodomethyl concentration is estimated to be $1.8 \times 10^{12} \mathrm{~cm}^{-3}$. With increasing $\left[\mathrm{O}_{2}\right]$, the decay rate of the $\mathrm{CH}_{2} \mathrm{I}$ increased monotonically, and the pseudo first-order rate coefficient, $k^{\prime}$ $=k \times\left[\mathrm{O}_{2}\right]$, was measured as a function of oxygen concentration [Figs. 6(b)-6(d)]. Pseudo first-order conditions were maintained with $\left[\mathrm{O}_{2}\right]$ being always at least a factor of 20 
(a)

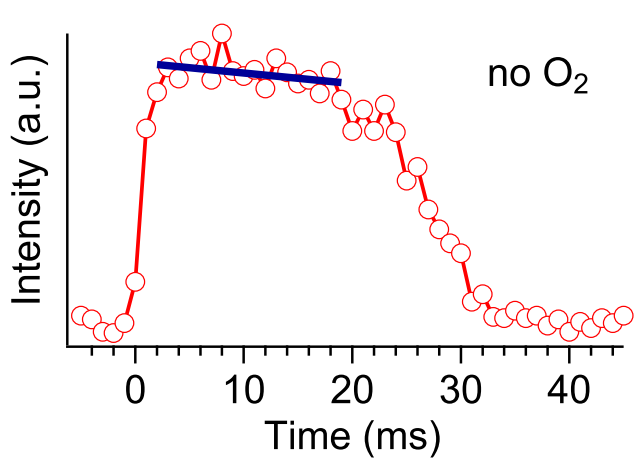

(c)

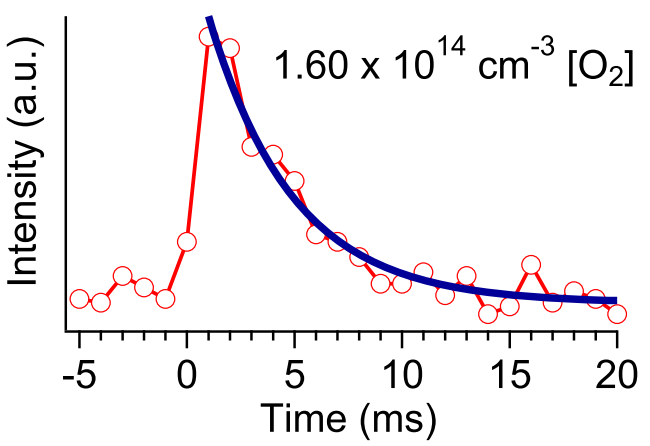

(b)

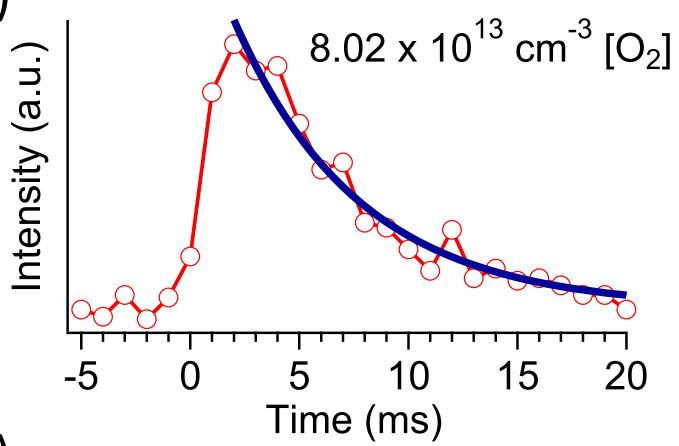

(d)

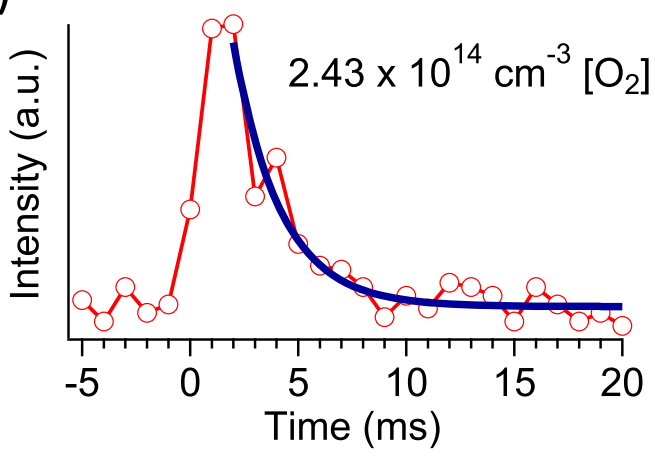

(e)

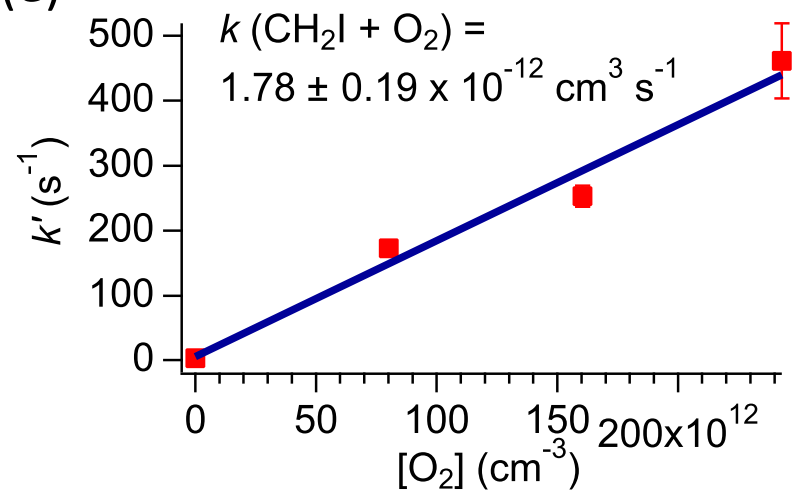

FIG. 6. Kinetic time dependent $\mathrm{CH}_{2} \mathrm{I}$ signal is shown in [(a)-(d)] at various $\mathrm{O}_{2}$ concentrations. Solid lines represent single exponential fits to the experimental kinetic traces, from which pseudo-first order rate constants are derived. (e) Dependence of the pseudo-first-order $\mathrm{CH}_{2} \mathrm{I}$ decay constant on the concentration of $\mathrm{O}_{2}$. The second-order rate coefficient is given by the slope of the linear fit.

larger than $\left[\mathrm{CH}_{2} \mathrm{I}\right]$. The only significant process consuming $\mathrm{CH}_{2} \mathrm{I}$ radicals was the reaction with $\mathrm{O}_{2}$. The bimolecular rate coefficient could be obtained from the slope of the $k^{\prime}$ vs. $\left[\mathrm{O}_{2}\right]$ plot [Fig. 6(e)]. Our measured room temperature second-order rate coefficient $k\left(\mathrm{CH}_{2} \mathrm{I}+\mathrm{O}_{2}\right)=(1.78 \pm 0.19) \times 10^{-12} \mathrm{~cm}^{3} \mathrm{~s}^{-1}$ is in good agreement with the literature values, which are in the range of $1.28-1.82 \times 10^{-12} \mathrm{~cm}^{3} \mathrm{~s}^{-1}$. ${ }^{56-62}$

As described in Sec. II, electron-ion coincidence events can be defined post-measurement by selecting regions of interest on both position sensitive detectors as well as choosing a kinetic time interval measured from the laser trigger signal. These constraints create a time series of ion mass spectra within a defined velocity range of the neutrals, in coincidence with photoelectrons of a chosen kinetic energy range (for example, threshold electrons). The $\mathrm{CH}_{2} \mathrm{I}+\mathrm{O}_{2}$ reaction studied in this section produces the transient species $\mathrm{CH}_{2} \mathrm{OO}$, the smallest Criegee intermediate. The signal from miniscule concentrations of such fleeting species can be enhanced by integrating only coincidence events with appropriate detector spatial constraints and within a chosen kinetic time interval, as shown in Fig. 7.

\section{Measuring unimolecular dissociation rate constants}

Finally, to illustrate the capability of the experiment to measure unimolecular dissociation rate constants, we show two time-of-flight distributions of iodocyclohexane in Fig. 8, taken at a photon energy of $11.55 \mathrm{eV}$ using a room temperature effusive sample source and a $125 \mathrm{~V} \mathrm{~cm}^{-1}$ extraction field. To construct the time-of-flight spectrum in Fig. 8(a), a circular photoelectron region of interest is set such that all electrons with less than $100 \mathrm{meV}$ kinetic energy are considered. With increasing photoelectron kinetic energy, a progressively smaller ratio of the Newton sphere's projection falls within this region. These "hot" electrons are released with kinetic energy, which decreases the internal energy of the parent 
(a)

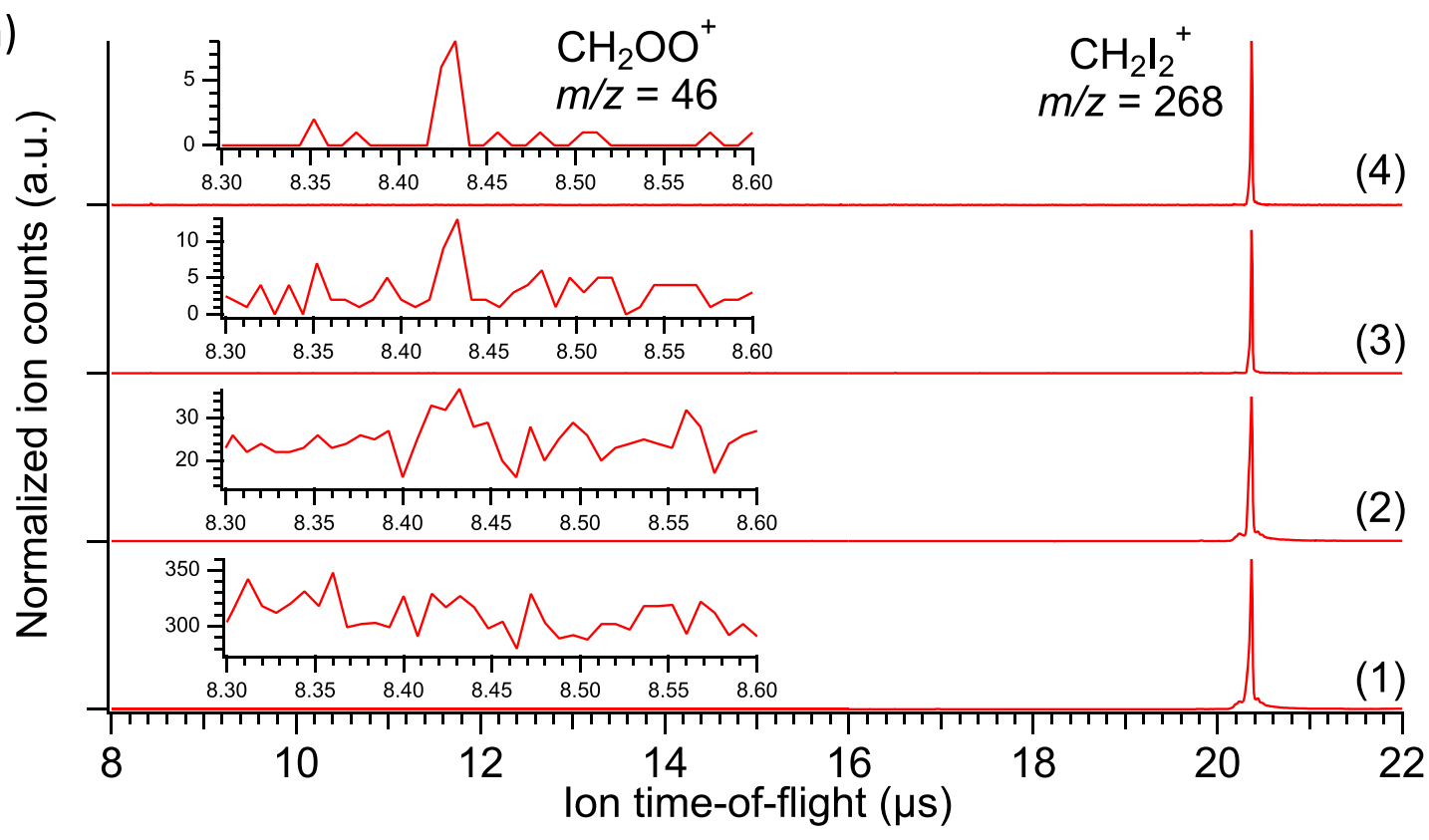

(b)

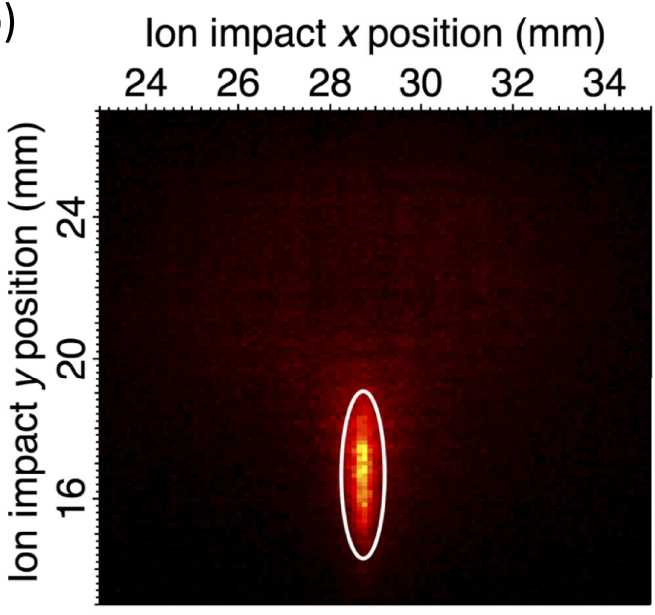

(c)

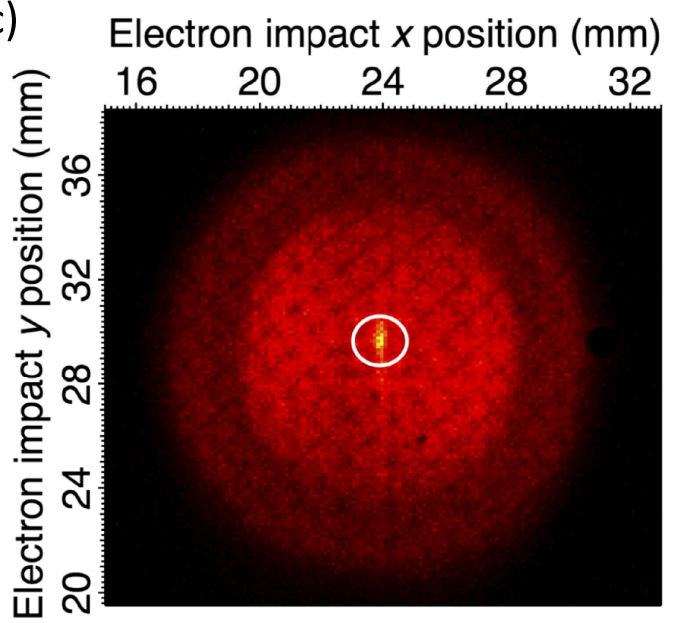

FIG. 7. (a) Typical time-of-flight mass spectra of the $\mathrm{CH}_{2} \mathrm{I}+\mathrm{O}_{2}$ reaction, with velocity map images of ions (b), and electrons (c). The mass spectra in (a) are derived using (1) the whole ion and electron images with no kinetic time restriction; (2) selecting the region of interest in the electron image (shown by the circle) to only low-energy electrons; (3) selecting the regions of interest in both the electron and ion images; (4) selecting the regions of interest in both images, and restricting the analysis to a kinetic time of $0-5 \mathrm{~ms}$ after the laser pulse.

ion available for dissociation, and some will be sufficiently stabilized to be detected as $\mathrm{C}_{6} \mathrm{H}_{11} \mathrm{I}^{+}$in the spectrum of Fig. 8(a). Including only threshold electrons as the TOF start signal $^{25}$ yields the TOF trace in Fig. 8(b). In this case, the room temperature internal energy of the sample and $100 \%$ of the energy of the photon above the ionization energy are available for dissociation, which is enough to quantitatively and promptly eject the halogen atom from the parent ion. The majority of the cyclohexyl ions formed have enough internal energy to further dissociate, ejecting ethylene to form $\mathrm{C}_{4} \mathrm{H}_{7}{ }^{+}$. This process is slow at energies close to threshold, and cyclohexyl ions continue to dissociate while inside the ion optics. These dissociation events yield daughter ions that are slower than promptly formed $\mathrm{C}_{4} \mathrm{H}_{7}{ }^{+}$, but faster than their parent $\mathrm{C}_{6} \mathrm{H}_{11}{ }^{+}$, resulting in a wide range of total flight times and in a quasi-exponentially decaying time-of-flight peak shape as observed above $17 \mu$ s. Such asymmetrically broadened daughter ion peak shapes can now be routinely modeled to extract unimolecular rate constants, and statistical theories are available to model and extrapolate the experimental rate curve to threshold, thereby taking kinetic shifts quantitatively into account. ${ }^{63}$ Correcting for the kinetic shift is imperative to derive accurate dissociative photoionization onsets and, through them, ionic bond energies. Slow fragmentation processes can also help unveil the dissociation mechanism. For instance, the fact that the $\mathrm{C}_{4} \mathrm{H}_{7}{ }^{+}$peak is broadened establishes it as a product of a sequential channel because slow $\mathrm{C}_{2} \mathrm{H}_{4} \mathrm{I}$ loss from the parent ion could not compete with fast I atom loss. As an added benefit, metastable peak profiles indicate unambiguously that the ion in question is formed in dissociative photoionization as opposed to fragmentationfree photoionization of a neutral fragment of the precursor molecule. In analyzing complex reactive systems, such information can also aid signal apportioning and may be necessary for deriving quantitative branching fractions of competing processes. $^{29}$ 


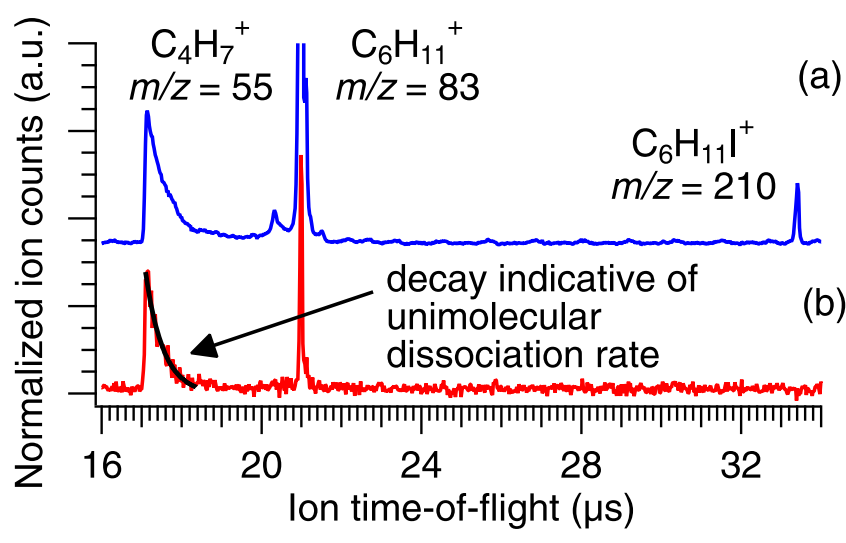

FIG. 8. Photoionization mass spectrum of iodocyclohexane at a photon energy of $11.55 \mathrm{eV}$, in coincidence with (a) mostly slow electrons within a $100 \mathrm{meV}$ circle on the VMI image and (b) only threshold electrons. In spectrum (a) (contaminated with hot electrons), the undissociated iodocyclohexane ion, $\mathrm{C}_{6} \mathrm{H}_{11} \mathrm{I}^{+}$, is seen together with two fragment ions: the I-loss product cyclohexyl ion, $\mathrm{C}_{6} \mathrm{H}_{11}{ }^{+}$, as well as the product of its sequential ethylene loss, $\mathrm{C}_{4} \mathrm{H}_{7}{ }^{+}$.

\section{CONCLUSIONS}

The new prototype CRF-PEPICO apparatus applies recent advances in continuous PEPICO spectroscopy to kinetically resolved studies of reactive gas mixtures. It successfully combines a slow-flow laser photolysis inlet system with electron and ion optics that simultaneously achieve velocity map imaging and Wiley-McLaren space focusing of cations. This advance allows us to record high resolution mass spectra and photoion momentum images in coincidence with velocity map imaged photoelectrons. We do this by spatially separating the axial extraction and lateral velocity map imaging fields using many-electrode electron and ion optics. Sharp peaks in the time-of-flight coincidence spectra inherently result in a high signal-to-noise ratio with respect to the false coincidence background, but the dynamic range of the experiment can further be increased by applying the temporal ion deflection scheme, as discussed previously. ${ }^{29}$ Furthermore, the possibility to have a long, uniform ion extraction field, which is reconfigurable without breaking vacuum, means that rate constants for metastable cation dissociations can now be measured in a PEPICO double VMI apparatus.

To demonstrate the capabilities of the new design, we used laser photolysis to create propargyl and iodomethyl radicals in a collisional environment. The photoion mass-selected threshold photoelectron spectra of these two radicals agree well with the literature spectra, and the thermalized iodomethyl radical source offered new insights into the vibrational structure of the TPES, only observable in room temperature or colder samples. We validated the reaction kinetics performance of the spectrometer by measuring a bimolecular rate constant of the $\mathrm{CH}_{2} \mathrm{I}+\mathrm{O}_{2}$ reaction that agrees with the accepted literature value and observed the smallest Criegee intermediate, $\mathrm{CH}_{2} \mathrm{OO}$.

Finally, to illustrate the capability of the CRF-PEPICO experiment to measure unimolecular dissociation rate constants, we have shown time-of-flight distribution functions of iodocyclohexane ion dissociation that are indicative of slow (metastable) dissociation of the cyclohexyl ion.

\section{ACKNOWLEDGMENTS}

The authors thank Dr. Oliver Welz for his contributions to the $\mathrm{CH}_{2} \mathrm{I}+\mathrm{O}_{2}$ experiments. This work has been funded by the National Science Foundation (Grant No. CHE-1266407), by the U.S. Department of Energy under the Visiting Faculty Program, and by the Swiss Federal Office for Energy (BFE Contract No. SI/501269-01). This material is based upon work supported by the U.S. Department of Energy, Office of Science, Office of Basic Energy Sciences.

${ }^{1}$ R. K. Yoo, B. Ruscic, and J. Berkowitz, J. Chem. Phys. 96, 911 (1992).

${ }^{2}$ X. Yang, J. Lin, Y. T. Lee, D. A. Blank, A. G. Suits, and A. M. Wodtke, Rev. Sci. Instrum. 68, 3317 (1997).

${ }^{3}$ J. C. Traeger, Int. J. Mass Spectrom. 194, 261 (2000).

${ }^{4}$ F. Qi, R. Yang, B. Yang, C. Q. Huang, L. X. Wei, J. Wang, L. S. Sheng, and Y. W. Zhang, Rev. Sci. Instrum. 77, 84101 (2006).

${ }^{5}$ D. L. Osborn, P. Zou, H. Johnsen, C. C. Hayden, C. A. Taatjes, V. D. Knyazev, S. W. North, D. S. Peterka, M. Ahmed, and S. R. Leone, Rev. Sci. Instrum. 79, 104103 (2008).

${ }^{6}$ C. A. Taatjes, N. Hansen, D. L. Osborn, K. Kohse-Hoinghaus, T. A. Cool, and P. R. Westmoreland, Phys. Chem. Chem. Phys. 10, 20 (2008).

${ }^{7}$ O. Kostko, B. Bandyopadhyay, and M. Ahmed, Annu. Rev. Phys. Chem. 67, 19 (2016).

${ }^{8}$ J. D. Savee, E. Papajak, B. Rotavera, H. Huang, A. J. Eskola, O. Welz, L. Sheps, C. A. Taatjes, J. Zador, and D. L. Osborn, Science 347, 643 (2015).

${ }^{9}$ J. Zádor, H. Huang, O. Welz, J. Zetterberg, D. L. Osborn, and C. A. Taatjes, Phys. Chem. Chem. Phys. 15, 10753 (2013).

${ }^{10}$ C. A. Taatjes, G. Meloni, T. M. Selby, A. J. Trevitt, D. L. Osborn, C. J. Percival, and D. E. Shallcross, J. Am. Chem. Soc. 130, 11883 (2008).

${ }^{11}$ O. Welz, J. D. Savee, D. L. Osborn, S. S. Vasu, C. J. Percival, D. E. Shallcross, and C. A. Taatjes, Science 335, 204 (2012).

${ }^{12}$ M. Capron, J. Bourgalais, R. K. Abhinavam Kailasanathan, D. L. Osborn, S. D. Le Picard, and F. Goulay, Phys. Chem. Chem. Phys. 17, 23833 (2015).

${ }^{13}$ J. Bourgalais, M. Capron, R. K. A. Kailasanathan, D. L. Osborn, K. M. Hickson, J.-C. Loison, V. Wakelam, F. Goulay, and S. D. Le Picard, Astrophys. J. 812, 106 (2015).

${ }^{14}$ J. Bourgalais, M. Spencer, D. L. Osborn, F. Goulay, and S. D. Le Picard, J. Phys. Chem. A 120, 9138 (2016).

${ }^{15}$ A. J. Trevitt, F. Goulay, G. Meloni, D. L. Osborn, C. A. Taatjes, and S. R. Leone, Int. J. Mass Spectrom. 280, 113 (2009).

${ }^{16}$ A. Bodi, P. Hemberger, D. L. Osborn, and B. Sztáray, J. Phys. Chem. Lett. 4, 2948 (2013).

${ }^{17}$ I. Fischer, T. Schüßler, H.-J. Deyerl, M. Elhanine, and C. Alcaraz, Int. J. Mass Spectrom. 261, 227 (2007).

${ }^{18}$ F. Holzmeier, M. Lang, P. Hemberger, A. Bodi, M. Schäfer, R. D. Dewhurst, H. Braunschweig, and I. Fischer, Chem. - Eur. J. 20, 9683 (2014).

${ }^{19}$ P. Hemberger, A. J. Trevitt, T. Gerber, E. Ross, and G. da Silva, J. Phys. Chem. A 118, 3593 (2014).

${ }^{20}$ S. Liang, P. Hemberger, N. M. Neisius, A. Bodi, H. H. Grützmacher, J. Levalois-Grützmacher, and S. Gaan, Chem. - Eur. J. 21, 1073 (2015).

${ }^{21}$ J. Bouwman, A. Bodi, J. Oomens, and P. Hemberger, Phys. Chem. Chem. Phys. 17, 20508 (2015).

${ }^{22}$ G. T. Buckingham, T. K. Ormond, J. P. Porterfield, P. Hemberger, O. Kostko, M. Ahmed, D. J. Robichaud, M. R. Nimlos, J. W. Daily, and G. B. Ellison, J. Chem. Phys. 142, 044307 (2015).

${ }^{23}$ T. Baer and R. P. Tuckett, Phys. Chem. Chem. Phys. 19, 9698 (2017).

${ }^{24}$ D. H. Parker and A. T. J. B. Eppink, J. Chem. Phys. 107, 2357 (1997).

${ }^{25}$ B. Sztáray and T. Baer, Rev. Sci. Instrum. 74, 3763 (2003).

${ }^{26}$ A. Bodi, M. Johnson, T. Gerber, Z. Gengeliczki, B. Sztáray, and T. Baer, Rev. Sci. Instrum. 80, 034101 (2009).

${ }^{27}$ G. A. Garcia, H. Soldi-Lose, and L. Nahon, Rev. Sci. Instrum. 80, 023102 (2009).

${ }^{28}$ A. Bodi, B. Sztáray, T. Baer, M. Johnson, and T. Gerber, Rev. Sci. Instrum. 78, 084102 (2007).

${ }^{29}$ D. L. Osborn, C. C. Hayden, P. Hemberger, A. Bodi, K. Voronova, and B. Sztáray, J. Chem. Phys. 145, 164202 (2016).

${ }^{30}$ J. D. Savee, S. Soorkia, O. Welz, T. M. Selby, C. A. Taatjes, and D. L. Osborn, J. Chem. Phys. 136, 134307 (2012). 
${ }^{31}$ J. D. Savee, O. Welz, C. A. Taatjes, and D. L. Osborn, Phys. Chem. Chem. Phys. 14, 10410 (2012).

${ }^{32}$ X. Tang, X. Zhou, M. Niu, S. Liu, J. Sun, X. Shan, F. Liu, and L. Sheng, Rev. Sci. Instrum. 80, 113101 (2009).

${ }^{33}$ G. A. Garcia, B. K. Cunha de Miranda, M. Tia, S. Daly, and L. Nahon, Rev. Sci. Instrum. 84, 053112 (2013).

${ }^{34}$ X. Tang, G. A. Garcia, J.-F. Gil, and L. Nahon, Rev. Sci. Instrum. 86, 123108 (2015).

${ }^{35}$ A. Bodi, P. Hemberger, T. Gerber, and B. Sztáray, Rev. Sci. Instrum. 83, 083105 (2012).

${ }^{36}$ A. Bodi and P. Hemberger, Phys. Chem. Chem. Phys. 16, 505 (2014).

${ }^{37}$ W. C. Wiley and I. H. McLaren, Rev. Sci. Instrum. 26, 1150 (1955).

${ }^{38}$ I. R. Slagle, F. Yamada, and D. Gutman, J. Am. Chem. Soc. 103, 149 (1981).

${ }^{39}$ M. Ryazanov and H. Reisler, J. Chem. Phys. 138, 144201 (2013).

${ }^{40}$ J. A. Miller and C. F. Melius, Combust. Flame 91, 21 (1992).

${ }^{41}$ P.-T. Howe and A. Fahr, J. Phys. Chem. A 107, 9603 (2003).

${ }^{42}$ J. D. DeSain and C. A. Taatjes, J. Phys. Chem. A 107, 4843 (2003).

${ }^{43}$ J. A. Miller and S. J. Klippenstein, J. Phys. Chem. A 105, 7254 (2001).

${ }^{44}$ J. A. Miller and S. J. Klippenstein, J. Phys. Chem. A 107, 7783 (2003).

${ }^{45}$ H. Xu and S. T. Pratt, J. Phys. Chem. A 117, 9331 (2013).

${ }^{46}$ H.-J. Deyerl, I. Fischer, and P. Chen, J. Chem. Phys. 111, 3441 (1999).

${ }^{47}$ P. Hemberger, M. Lang, B. Noller, I. Fischer, C. Alcaraz, K. C. De Miranda, and G. A. Garcia, J. Phys. Chem. A 115, 2225 (2011).

${ }^{48}$ B. P. Tsai, A. S. Werner, and T. Baer, J. Chem. Phys. 63, 4384 (1975).

${ }^{49}$ W. A. Chupka, J. Chem. Phys. 98, 4520 (1993).

${ }^{50}$ U. Jacovella, B. Gans, and F. Merkt, J. Chem. Phys. 139, 084308 (2013).
${ }^{51}$ A. Saiz-lopez, J. M. C. Plane, A. R. Baker, L. J. Carpenter, R. Von Glasow, C. G. Juan, G. Mcfiggans, and R. W. Saunders, Chem. Rev. 112, 1773 (2012).

${ }^{52}$ J. L. L. Jimenez, R. Bahreini, D. R. Cocker III, G. Zhuang, V. Varutbangkul, R. C. Flagan, J. H. Seinfeld, C. D. O'Dowd, and T. Hoffmann, J. Geophys. Res., [Atmos.] 108, D10, doi:10.1029/2002JD002452 (2003).

${ }^{53}$ L. Andrews, J. M. Dyke, N. Jonathan, N. Keddar, and A. Morris, J. Phys. Chem. 88, 1950 (1984).

${ }^{54}$ V. A. Mozhayskiy and A. I. Krylov, ezSpectrum, http://iopenshell.usc. edu/downloads, 2016.

${ }^{55}$ J. Sehested, T. Ellermann, and O. J. Nielsen, Int. J. Chem. Kinet. 26, 259 (1994).

${ }^{56}$ W.-L. Ting, C.-H. Chang, Y.-F. Y.-P. Lee, H. Matsui, Y.-F. Y.-P. Lee, and J. J.-M. Lin, J. Chem. Phys. 141, 104308 (2014).

${ }^{57}$ L. Sheps, J. Phys. Chem. Lett. 4, 4201 (2013).

${ }^{58}$ H. Huang, A. J. Eskola, and C. A. Taatjes, J. Phys. Chem. Lett. 3, 3399 (2012).

${ }^{59}$ A. J. Eskola, D. Wojcik-Pastuszka, E. Ratajczak, and R. S. Timonen, Phys. Chem. Chem. Phys. 8, 1416 (2006).

${ }^{60}$ A. Masaki, S. Tsunashima, and N. Washida, J. Phys. Chem. 99, 13126 (1995).

${ }^{61}$ S. Enami, Y. Sakamoto, T. Yamanaka, S. Hashimoto, M. Kawasaki, K. Tonokura, and H. Tachikawa, Bull. Chem. Soc. Jpn. 81, 1250 (2008).

${ }^{62}$ D. Stone, M. Blitz, L. Daubney, T. Ingham, and P. Seakins, Phys. Chem. Chem. Phys. 15, 19119 (2013).

${ }^{63}$ B. Sztáray, A. Bodi, and T. Baer, J. Mass Spectrom. 45, 1233 (2010). 